\title{
Clinical Outcomes of Totally Laparoscopic Total Gastrectomy versus Open Total Gastrectomy for Remnant Gastric Cancer
}

\author{
Su Jung Choi, M.D. ${ }^{1}$, Chung Sik Gong, M.D. ${ }^{2}$, Byung Sik Kim, M.D., Ph.D. ${ }^{2}$, Seon Ok Kim, M.Sc. ${ }^{3}$, Hee Sung Kim, M.D., Ph.D. ${ }^{2}$ \\ 'Department of Surgery, ${ }^{2}$ Department of Gastric Surgery, and ${ }^{3}$ Department of Clinical Epidemiology and Biostatistics, Asan Medical Center, University of \\ Ulsan College of Medicine, Seoul, Korea
}

Purpose: This study compares the feasibility and safety of Totally Laparoscopic Total Gastrectomy (TLTG) with Open Total Gastrectomy (OTG) for Remnant Gastric Cancer (RGC) in patients who had previously undergone gastrectomy.

Methods: We retrospectively collected and analyzed the data of 139 consecutive patients who underwent OTG along with 21 patients who underwent TLTG for RGC between January 2008 and December 2016. One-to-two Propensity Score Matching (PSM) was performed to compare the age, gender, body mass index, American Society of Anesthesiologists score, clinical tumor stage, previous gastric disease, previous gastrectomy type, previous reconstruction type, history of previous upper abdominal surgery except gastrectomy, and combined major operations. A total of sixty patients (21 who underwent TLTG and 39 who underwent OTG) were matched, and surgical outcomes and survival rates were compared.

Results: The TLTG patients were found to recover bowel movements sooner than the OTG group (OTG $3.74 \pm 0.88$ vs TLTG $3.19 \pm 0.81$ days, $p=0.02$ ). Post-operative surgical outcomes, including pathological features, clinical courses, complications and survival rates did not differ between the two groups ( $p>0.05)$.

Conclusion: Although TLTG was not found to have any definitive clinical advantage over OTG except for more rapid recovery of bowel movement, TLTG should be considered as safe and feasible surgical procedure as OTG for the treatment of RGC.

Keywords: Laparoscopy, Total gastrectomy, Gastric cancer, Remnant cancer
Received August 1, 2018

Revised 1st September 27, 2018

2nd October 26, 2018

3rd October 29, 2018

Accepted October 30, 2018

Corresponding author

Hee Sung Kim

Department of Gastric Surgery, Asan Medical Center, University of Ulsan College of Medicine, 88 Olympic-ro

43-gil, Songpa-gu, Seoul 05505, Korea Tel: +82-2-3010-1509

Fax: +82-2-474-9027

E-mail: os-khs@hanmail.net ORCID:

http://orcid.org/0000-0003-2351-4402
This is an Open Access article distributed under the terms of the Creative Commons Attribution Non-Commercial License (http:// creativecommons.org/licenses/by-nc/4.0/) which permits unrestricted non-commercial use, distribution, and reproduction in any medium, provided the original work is properly cited. Copyright @ 2019 The Journal of Minimally
Invasive Surgery. All rights reserved.

\section{INTRODUCTION}

$\mathrm{RGC}$ is defined as gastric cancer which develops in the remnant stomach after subtotal gastrectomy for benign disease or gastric cancer., ${ }^{1,2}$ It is rare, and has been reported to account for 1 8\% of all gastric cancers. ${ }^{3,4}$ The incidence of early stage RGC has increased because of early detection resulting from periodic endoscopic surveillance after gastrectomy, and offers the opportunity to be managed by a minimally invasive pro- cedure. ${ }^{5}$ LG is widely accepted, but is less frequently used to treat RGC because of technical difficulties, concerns over radical resection, and long operation time. LG in RGC has been successfully performed by experienced laparoscopic surgeons since Yamada et al. ${ }^{6}$ first described its use in early stage RGC patients. We have reported $L G$ is a valid surgical treatment for RGC with 17 cases of all LG including 6 cases of TLTG for $\mathrm{RGC}$ in previous study. In this study, the safety and feasibility of TLTG for RGC were evaluated and compared with OTG. 
Due to recent advancements in laparoscopic instrumentation, surgical techniques, and surgeons' experience, the spectrum of diseases that can be treated by means of complex procedures such as laparoscopic functional preservation surgery and laparoscopic total gastrectomy has expanded. As a result, laparoscopic gastrectomy for gastric cancer has received wider clinical acceptance. Despite its significant advantages, Laparoscopic gastrectomy (LG) has been carried out less frequently in the treatment of remnant gastric cancer (RGC) due to the technical challenges posed by intraperitoneal adhesions, anatomic displacements, and concerns for radical resection in patients with previous gastrectomies. ${ }^{1,8}$ Adhesions are responsible for extending operative time and increasing the risk of organ injury, and the evidence for oncologic safety of laparoscopic procedures in RGC is scarce. Nevertheless, surgeons are broadening the spectrum of laparoscopic procedures and LG is now possible in patients who have previously undergone gastrectomy.

The short- and long-term surgical outcomes of LG for RGC have only been investigated a limited number of patients who previously underwent gastrectomies, and no efficacy study has been reported for Totally Laparoscopic Total Gastrectomy (TLTG) for RGC. Our institution has reported success with LG for $\mathrm{RGC}^{7}$ and now we report on a comparison between the efficacies of TLTG and Open Total Gastrectomy (OTG) for gastric cancer patients with RGC who had previously undergone gastrectomies. Our aim was to evaluate the safety and feasibility as well as the non-inferiority of TLTG compared with OTG for RGC.

\section{MATERIALS AND METHODS}

\section{Patients}

We retrospectively reviewed the data of 160 consecutive patients who underwent total gastrectomy for gastric cancer between January 2008 and December 2016 in Asan Medical Center. Of the 160 patients, 139 underwent OTG and 21 underwent TLTG. The diagnosis was based on preoperative examinations including esophagogastroduodenoscopy, endoscopic ultrasound, and computed tomography. The patient inclusion criteria were as follows: (1) patients who underwent previous gastrectomy due to gastric cancer or gastric ulcer, (2) lesions pathologically confirmed as gastric cancer, (3) no distant metastasis, and (4) a total gastrectomy was performed. The patient exclusion criteria were as follows: (1) open conversion cases from the laparoscopic approach due to serosaexposed advanced gastric cancer (AGC), (2) palliative surgery, and (3) emergency surgery from bleeding.

\section{TLTG technique}

Adhesiolysis around the upper abdominal wall and connective tissue was necessary in all cases. In sixty-eight patients with previous Gastroduodenostomies (GD), adhesions around the GD area were carefully dissected, and the duodenum was transected just below the duodenal bulb using an endoscopic linear stapler (ECHELON FLEX ${ }^{\mathrm{TM}}$ 60, gold cartridge, Ethicon Endo-Surgery, Inc., Cincinnati, OH, USA) with a closed staple of $1.8 \mathrm{~mm}$ in height. In ninety-two cases with previous Gastrojejunostomies (GJ) the site was isolated, and the afferent and efferent jejunal loops were transected. Then, the remaining lymph nodes around the common hepatic, proximal or distal splenic, celiac, and left gastric arteries were dissected (in that order), followed by the remnant lymph nodes such as 2 , 4sa, 10, and the mesenteric lymph nodes. The remnant stomach and pancreas were separated from the adherent surface of the liver. This procedure was carefully performed using ultrasonic shears and laparoscopic blunt scissors. Finally, the remnant stomach and gastroesophageal junction was completely mobilized. After the resected specimen was retrieved through the extended umbilical port site, an intracorporeal Esophagojejunostomy (EJ) was performed using the endoscopic linear stapler with a closed staple height of $1.5 \mathrm{~mm}$. Finally, defects in Peterson's space and the trans-mesenteric space were closed with sutures. This procedure has been described in detail. ${ }^{9-11}$

\section{Clinical evaluation of surgical outcomes}

The data obtained from the medical records included information referring to the age, gender, Body Mass Index (BMI), American Society of Anesthesiologists (ASA) score, history of previous abdominal surgery, operative time, change of preand post-operative hemoglobin and hematocrit, time to first flatus, pain score by Visual Analogue Scale (VSA), number of analgesics administered, intra and post-operative transfusion, intra-operative events, post-operative hospital stay, tumor size, number of retrieved lymph nodes, resection margins and pathologic TNM stage of the patients. Combined major operations were pancreas, bile duct and colorectal cancer surgeries but not appendectomy, cholecystectomy, and splenectomy. Intra-operative events included emphysema, injury to organs such as pancreas, spleen, colon, small bowel, liver and major vessels, and anastomosis site events. Intra-operative anastomosis events referred to all unexpected events related to the EJ anastomosis, such as tearing and kinking at the anastomotic site of due to failure of reconstruction, pseudo-lumen stapling, and compression of the crus muscle, etc. Post-operative pain control consisted of intravenous, patient-controlled analgesia (fentanyl $2500 \mu \mathrm{g}$, ketorolac tromethamine $180 \mathrm{mg}$, 
and ondansetron hydrochloride $16 \mathrm{mg}$ ), and intermittent analgesic infusions. Post-operative pain was assessed by VSA and by the number of the additional doses of analgesics required during post-operative hospital stay. A post-operative complication was defined as any event that required conservative or surgical treatment after surgery. Early complications were defined as events occurring within 30 days of surgery, and late complications as those occurring after that. Complications were reviewed and classified according to the Clavien-Dindo Classification (CDC) system. ${ }^{12}$

\section{Statistical analysis}

The data were analyzed using SPSS version 18.0 (SPSS Inc.,

Table 1. Clinical characteristics of patients who underwent OTG and TLTG

\begin{tabular}{|c|c|c|c|c|c|c|c|}
\hline \multirow{2}{*}{ Variable } & \multicolumn{2}{|c|}{ Total set $(n=160)$} & \multirow{2}{*}{$p$ value } & \multirow{2}{*}{ Stddiff } & \multicolumn{2}{|c|}{ PS matched set $(1: 2)(n=60)$} & \multirow{2}{*}{ Stddif } \\
\hline & OTG $(n=139)$ & TLTG $(n=21)$ & & & OTG $(n=39)$ & TLTG $(n=21)$ & \\
\hline Age (years, mean \pm SD) & $60.56 \pm 11.14$ & $61.10 \pm 13.38$ & 0.842 & 0.040 & $61.38 \pm 10.18$ & $61.10 \pm 13.38$ & 0.022 \\
\hline Gender & & & 0.352 & 0.248 & & & 0.037 \\
\hline Male & $99(71.22)$ & $17(80.95)$ & & & $31(79.49)$ & $17(80.95)$ & \\
\hline Female & $40(28.78)$ & $4(19.05)$ & & & $8(20.51)$ & 4 (19.05) & \\
\hline BMI & $21.53 \pm 2.78$ & $21.25 \pm 2.83$ & 0.669 & 0.098 & $21.32 \pm 2.3$ & $21.25 \pm 2.83$ & 0.026 \\
\hline ASA score & & & 0.377 & 0.297 & & & 0.056 \\
\hline I & 99 (71.22) & 12 (57.14) & & & 23 (58.97) & 12 (57.14) & \\
\hline$\|$ & 31 (22.3) & $7(33.33)$ & & & $12(30.77)$ & 7 (33.33) & \\
\hline III & $9(6.47)$ & $2(9.52)$ & & & $4(10.26)$ & $2(9.52)$ & \\
\hline Clinical tumor stage & & & 0.010 & 0.823 & & & 0.031 \\
\hline I & $70(50.36)$ & 18 (85.71) & & & $34(84.62)$ & $18(85.71)$ & \\
\hline$\|$ & $40(28.78)$ & $2(9.52)$ & & & $4(10.26)$ & $2(9.52)$ & \\
\hline III & $29(20.86)$ & $1(4.76)$ & & & $2(5.13)$ & $1(4.76)$ & \\
\hline Previous gastric disease & & & 0.130 & 0.455 & & & 0.031 \\
\hline Benign & 42 (30.22) & $3(14.29)$ & & & $6(15.38)$ & $3(14.29)$ & \\
\hline Malignancy & 97 (69.78) & $18(85.71)$ & & & 33 (84.62) & $18(85.71)$ & \\
\hline Previous gastrectomy type & & & 0.002 & 0.592 & & & 0.204 \\
\hline Open gastrectomy & 126 (90.65) & $13(61.9)$ & & & $28(71.79)$ & $13(61.9)$ & \\
\hline Laparoscopic gastrectomy & $13(9.35)$ & $8(38.1)$ & & & $11(28.21)$ & $8(38.1)$ & \\
\hline Previous recontruction type & & & 0.016 & 0.590 & & & 0.000 \\
\hline Gastroduodenostomy & $54(38.85)$ & 14 (66.67) & & & $26(66.67)$ & 14 (66.67) & \\
\hline Gastrojejunostomy & $85(61.15)$ & 7 (33.33) & & & 13 (33.33) & $7(33.33)$ & \\
\hline $\begin{array}{l}\text { History of abdominal surgery } \\
\text { excecpt gastrectomy }\end{array}$ & & & 0.702 & 0.317 & & & 0.103 \\
\hline None & 123 (88.49) & $20(95.24)$ & & & 38 (97.44) & $20(95.24)$ & \\
\hline Yes & $16(11.51)$ & $1(4.76)$ & & & $1(2.56)$ & $1(4.76)$ & \\
\hline Combined major operation & & & 0.045 & 0.646 & & & 0.000 \\
\hline None & 115 (82.73) & $21(100)$ & & & $39(100)$ & $21(100)$ & \\
\hline Yes & $24(17.27)$ & $0(0)$ & & & & & \\
\hline
\end{tabular}

Data are expressed as mean \pm standard deviation or number (percentage). OTG $=$ Open Total Gastrectomy; TLTG = Totally Laparoscopic Total Gastrectomy; PS = propensity score; Stddiff = standardized difference; BMI = body mass index; ASA = American Society of Anesthesiologi. 
Chicago, IL, USA) and SAS version 9.4 (SAS Institute, Cary, NC, USA). The chi-square test or Fisher's exact test were used to compare categorical variables and the Mann-Whitney U test was used to compare continuous variables. The differences in the risks of the binary and survival outcomes between the OTG and TLTG groups were assessed using univariate and multivariable logistic regression analyses and a Cox proportional hazards model, respectively. In the multivariable model, the variable selection was carried out using backward elimination. In order to reduce the impact of treatment-selection bias and of potential confounding factors in an observational study, we also performed Propensity Score Matching (PSM). The Propensity Scores (PS) were estimated by means of a multiple logistic regression analysis with the gastrectomy type as the dependent variable. A full non-parsimonious model was developed, which included age, gender, BMI, ASA, clinical tumor stage, previous gastric disease, previous gastrectomy type, previous reconstruction type, history of previous upper abdominal surgery except gastrectomy, and combined operations. We used the 1:2 ratio for Greedy matching with a caliper of 0.15 standard deviations of the logit of the estimated propensity score without replacement. Absolute standardized differences were used to diagnose the balance after the propensity analysis. In the propensity scorematched cohort, the risks of binary and survival outcomes were compared using generalized estimating equations and a Cox proportional hazards model with robust standard errors that accounted for the clustering of matched pairs. ${ }^{13}$ We furthermore adjusted for an absolute standardized difference greater than 0.15 . $p$ values $<0.05$ were considered statistically significant.

\section{RESULTS}

\section{Patient characteristics}

The clinicopathologic characteristic of the OTG and TLTG groups are shown in Table 1. Upon comparing all 160 patients,

Table 2. Pathologic results in the OTG and TLTG groups

\begin{tabular}{|c|c|c|c|c|c|c|}
\hline \multirow{2}{*}{ Variable } & \multicolumn{2}{|c|}{ Total set $(n=160)$} & \multirow{2}{*}{$p$ value } & \multicolumn{2}{|c|}{ PS-matched set (1:2) $(n=60)$} & \multirow{2}{*}{$p$ value } \\
\hline & OTG $(n=139)$ & TLTG $(n=21)$ & & OTG $(n=39)$ & TLTG $(n=21)$ & \\
\hline Tumor size & $4.65 \pm 3.25$ & $3.77 \pm 4.27$ & 0.021 & $3.14 \pm 1.93$ & $3.77 \pm 4.27$ & 0.539 \\
\hline Retrieved LNs & $13.08 \pm 10.78$ & $8.38 \pm 6.51$ & 0.102 & $9.92 \pm 11.75$ & $8.38 \pm 6.51$ & 0.576 \\
\hline Metastatic LNs & $0.73 \pm 1.86$ & $0.29 \pm 1.11$ & 0.120 & $0.26 \pm 0.82$ & $0.29 \pm 1.10$ & 0.739 \\
\hline PRM & $3.84 \pm 2.98$ & $2.05 \pm 1.76$ & 0.009 & $3.55 \pm 2.77$ & $2.05 \pm 1.76$ & 0.071 \\
\hline DRM & $6.61 \pm 5.94$ & $5.69 \pm 3.33$ & 0.861 & $7.11 \pm 6.59$ & $5.69 \pm 3.33$ & 0.898 \\
\hline T stage & & & 0.040 & & & 0.495 \\
\hline $\mathrm{T} 1$ & $54(38.85)$ & $13(61.9)$ & & $24(61.54)$ & $13(61.9)$ & \\
\hline $\mathrm{T} 2$ & $15(10.79)$ & $5(23.81)$ & & 6 (15.38) & $5(23.81)$ & \\
\hline Т3 & $30(21.58)$ & $1(4.76)$ & & $5(12.82)$ & $1(4.76)$ & \\
\hline T4 & 40 (28.78) & 2 (9.52) & & $4(10.26)$ & 2 (9.52) & \\
\hline $\mathrm{N}$ stage & & & 0.608 & & & 1.000 \\
\hline NO & $104(74.82)$ & $19(90.48)$ & & $34(87.18)$ & 19 (90.48) & \\
\hline N1 & $21(15.11)$ & $1(4.76)$ & & $3(7.69)$ & $1(4.76)$ & \\
\hline N2 & $10(7.19)$ & $1(4.76)$ & & $2(5.13)$ & $1(4.76)$ & \\
\hline N3 & $4(2.88)$ & $0(0)$ & & $0(0)$ & $0(0)$ & \\
\hline TNM stage & & & 0.047 & & & 0.895 \\
\hline I & 67 (48.2) & 17 (80.95) & & 30 (76.92) & 17 (80.95) & \\
\hline$\|$ & $40(28.78)$ & 2 (9.52) & & 6 (15.38) & 2 (9.52) & \\
\hline III & $31(22.3)$ & 2 (9.52) & & $3(7.69)$ & 2 (9.52) & \\
\hline IV & 1 (0.72) & $0(0)$ & & $0(0)$ & $0(0)$ & \\
\hline
\end{tabular}

Data are expressed as mean \pm standard deviation or number (percentage). OTG $=$ Open Total Gastrectomy; TLTG $=$ Totally Laparoscopic Total Gastrectomy; PS = Propensity Score; LN = lymph node; PRM = proximal resection margin; DRM = distal resection margin. 
the clinical tumor stage, previous gastrectomy type (open vs laparoscopic surgery), previous reconstruction type (GD vs GJ), and combined major operation differed significantly between the OTG and TLTG groups $(p<0.05)$. The advanced clinical tumor stage, previous OG, previous GJ reconstruction and combined major operation, such as pancreas, bile duct and colorectal surgeries, were more common in the OTG group, while most of the patients in the TLTG group were clinical stage I, and had previous LG, previous GD and no combined major operation. After the 1:2 PSM, 60 patients (21 who underwent TLTG and 39 who underwent OTG) were matched for the analysis, and all baseline variables included in the model were well-balanced within a standardized difference below 0.15 except for previous gastrectomy type (open vs laparoscopic surgery). We also adjusted the previous gastrectomy type with an absolute standardized difference of 0.204. The mean time intervals from the previous distal gastrectomy to the present total gastrectomy were $106.18 \pm 115.11$ months in the OTG group and $92.29 \pm 80.81$ months in the TLTG group after PSM, respectively $(p=0.745)$.

\section{Early surgical outcomes and post-operative clinical course after PSM}

Table 2 presents the postoperative pathologic results in the OTG and TLTG groups. The pathological characteristics did not differ significantly between the two groups thereafter. Data on early surgical outcomes and the postoperative clinical course are shown in Table 3. The mean operative time was

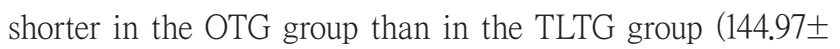

Table 3. Early surgical outcomes in the OTG and TLTG groups

\begin{tabular}{|c|c|c|c|c|c|c|}
\hline \multirow{2}{*}{ Variable } & \multicolumn{2}{|c|}{ Total set $(n=160)$} & \multirow{2}{*}{$p$ value } & \multicolumn{2}{|c|}{ PS matched set $(1: 2)(n=60)$} & \multirow{2}{*}{$p$ value } \\
\hline & OTG $(n=139)$ & TLTG $(n=21)$ & & OTG $(n=39)$ & TLTG $(n=21)$ & \\
\hline Operative time (min) & $156.30 \pm 43.95$ & $191.71 \pm 54.70$ & 0.004 & $144.97 \pm 30.41$ & $191.71 \pm 54.70$ & 0.001 \\
\hline Time to first flatus (days) & $3.98 \pm 1.05$ & $3.19 \pm 0.81$ & 0.001 & $3.74 \pm 0.88$ & $3.19 \pm 0.81$ & 0.020 \\
\hline Hemoglobin change $(\%)$ & $1.01 \pm 1.28$ & $1.70 \pm 1.25$ & 0.024 & $1.51 \pm 1.19$ & $1.70 \pm 1.25$ & 0.569 \\
\hline Hematocrit change $(\%)$ & $3.32 \pm 3.73$ & $4.97 \pm 3.95$ & 0.063 & $4.72 \pm 3.68$ & $4.97 \pm 3.95$ & 0.811 \\
\hline Intra-operative events (n) & & & 0.542 & & & 0.255 \\
\hline None & $115(82.73)$ & $16(76.19)$ & & $35(89.74)$ & 16 (76.19) & \\
\hline Yes & $24(17.24)$ & $5(23.81)$ & & $4(10.26)$ & $5(23.81)$ & \\
\hline Intra-operative anastomosis events (n) & & & 1.000 & & & 1.000 \\
\hline None & $137(98.56)$ & $21(100)$ & & $38(97.44)$ & $21(100)$ & \\
\hline Yes & $2(1.44)$ & $0(0)$ & & $1(2.56)$ & $0(0)$ & \\
\hline Intra-opreative transfusion (n) & & & 0.220 & & & - \\
\hline None & $126(90.65)$ & $21(100)$ & & $39(100)$ & $21(100)$ & \\
\hline Yes & $13(9.35)$ & $0(0)$ & & & & \\
\hline Post-operative transfusion (n) & & & 0.410 & & & 0.513 \\
\hline None & $105(75.54)$ & $18(85.71)$ & & 30 (76.92) & 18 (85.71) & \\
\hline Yes & $34(24.46)$ & $3(14.29)$ & & 9 (23.08) & 3 (14.29) & \\
\hline Pick of pain score & $7(6,10)$ & $6.5(6,7)$ & 0.154 & $7.5(6,9.5)$ & $6.6(6,7)$ & 0.260 \\
\hline Pain score at 8AM POD 1 & $3(3,4)$ & $3(3,4)$ & 0.110 & $3(3,4)$ & $3(3,4)$ & 0.331 \\
\hline Pain score at $8 \mathrm{AM}$ POD 3 & $3(2,3)$ & $3(2,3)$ & 0.823 & $3(2,3)$ & $3(2,3)$ & 0.707 \\
\hline Pain score at 8AM POD 5 & $2(1,3)$ & $2(2,2)$ & 0.247 & $2(1,3)$ & $2(2,2)$ & 0.617 \\
\hline Number of pain killer & $5(2,10)$ & $2(1,6)$ & 0.026 & $3(1,5)$ & $2(1,6)$ & 0.638 \\
\hline Post-operative hospital stay & $11(9,17)$ & $9(6,17)$ & 0.042 & $10(8,11)$ & $9(6,17)$ & 0.258 \\
\hline
\end{tabular}

Data are expressed as mean \pm standard deviation or number (percentage) or median (range). OTG $=$ Open Total Gastrectomy; TLTG $=$ Totally Laparoscopic Total Gastrectomy; PS = Propensity Score; POD = post operative day. 
30.41 vs $191.71 \pm 54.70 \mathrm{~min}$, respectively; $p=0.001$ ) and the time to first bowel movement was longer in the OTG group than in the TLTG group ( $3.74 \pm 0.88$ vs $3.19 \pm 0.81$ days; $p=0.020$ ). However, there were no significant changes in the pre- and post-operative serum hemoglobin and hematocrit level, intraoperative events, number of transfusions, pain score, numbers of administration of analgesics, and post-operative hospital stay $(p>0.05)$.

\section{Postoperative complications in the OTG and TLTG groups}

Early and late post-operative complications are showed in Table 4. There was no significant difference between the two groups in terms of early (OR 0.893, 95\% CI 0.337 2.368, $p=0.821)$ or late (OR $0.530,95 \%$ CI $0.064 \sim 4.384, p=0.556)$ complications in the total set. Likewise, after PSM, there were no significant differences between the two groups in terms of early (OR 1.540, 95\% CI 0.523 4.537, $p=0.433$ ) or late (OR 0.482, 95\% CI 0.047 4.939, $p=0.539$ ) complications (Table 5). The proportions of CDC category I, II, III and IV complications, whether early or late, were not significantly different $(p>0.05)$ between the two groups (Table 4). There were no postoperative deaths in either group.

\section{Overall Survivals (OS) and Disease-Free Survivals (DFS) in the OTG and TLTG groups}

The median follow-up period was 5.12 years (range 0.94 9.71 years). The 5-year OS rates of the OTG and TLTG

Table 4. Post-operative complications

\begin{tabular}{|c|c|c|c|c|c|c|}
\hline \multirow{2}{*}{ Variable } & \multicolumn{2}{|c|}{ Total Set $(n=160)$} & \multirow{2}{*}{$p$ value } & \multicolumn{2}{|c|}{ PS matched set $(1: 2)(n=60)$} & \multirow{2}{*}{$p$ value } \\
\hline & OTG $(n=139)$ & TLTG $(n=21)$ & & OTG $(n=39)$ & TLTG $(n=21)$ & \\
\hline \multicolumn{7}{|l|}{ Ealry Complications } \\
\hline Overall Complications & & & 0.534 & & & 0.432 \\
\hline None & 76 (54.68) & $13(61.9)$ & & $28(71.79)$ & $13(61.9)$ & \\
\hline Yes & $63(45.32)$ & $8(38.1)$ & & $11(28.21)$ & $8(38.1)$ & \\
\hline $\operatorname{CDC}$ & & & 0.946 & & & 0.543 \\
\hline 0 & $76(54.68)$ & $13(61.9)$ & & $28(71.79)$ & $13(61.9)$ & \\
\hline 1 & $6(4.32)$ & $1(4.76)$ & & $3(7.69)$ & $1(4.76)$ & \\
\hline 2 & $31(22.3)$ & $3(14.29)$ & & $5(12.82)$ & $3(14.29)$ & \\
\hline $3 \mathrm{~A}$ & $18(12.95)$ & $3(14.29)$ & & $1(2.56)$ & $3(14.29)$ & \\
\hline $3 B$ & $7(5.04)$ & $1(4.76)$ & & $2(5.13)$ & $1(4.76)$ & \\
\hline 4 & $1(0.72)$ & $0(0)$ & & $0(0)$ & $0(0)$ & \\
\hline \multicolumn{7}{|l|}{ Late Complications } \\
\hline Overall Complications & & & 0.702 & & & 0.649 \\
\hline None & $123(88.49)$ & $20(95.24)$ & & $35(89.74)$ & 20 (95.24) & \\
\hline Yes & $16(11.51)$ & $1(4.76)$ & & $4(10.26)$ & $1(4.76)$ & \\
\hline $\operatorname{CDC}$ & & & 1.000 & & & 0.789 \\
\hline 0 & 123 (88.49) & 20 (95.24) & & $35(89.24)$ & $20(95.24)$ & \\
\hline 1 & $2(1.44)$ & $0(0)$ & & $0(0)$ & $0(0)$ & \\
\hline 2 & $1(0.72)$ & $0(0)$ & & $0(0)$ & $0(0)$ & \\
\hline $3 \mathrm{~A}$ & $9(6.47)$ & $0(0)$ & & $0(0)$ & $0(0)$ & \\
\hline $3 B$ & 4 (2.88) & $0(0)$ & & $2(5.13)$ & $1(4.76)$ & \\
\hline 4 & $0(0)$ & $0(0)$ & & $2(5.13)$ & $0(0)$ & \\
\hline
\end{tabular}

Data are expressed as number (percentage). PS = Propensity Score; OTG = Open Total Gastrectomy; TLTG = Totally Laparoscopic Total Gastrectomy, CDC = Clavien-Deindo Classification. 


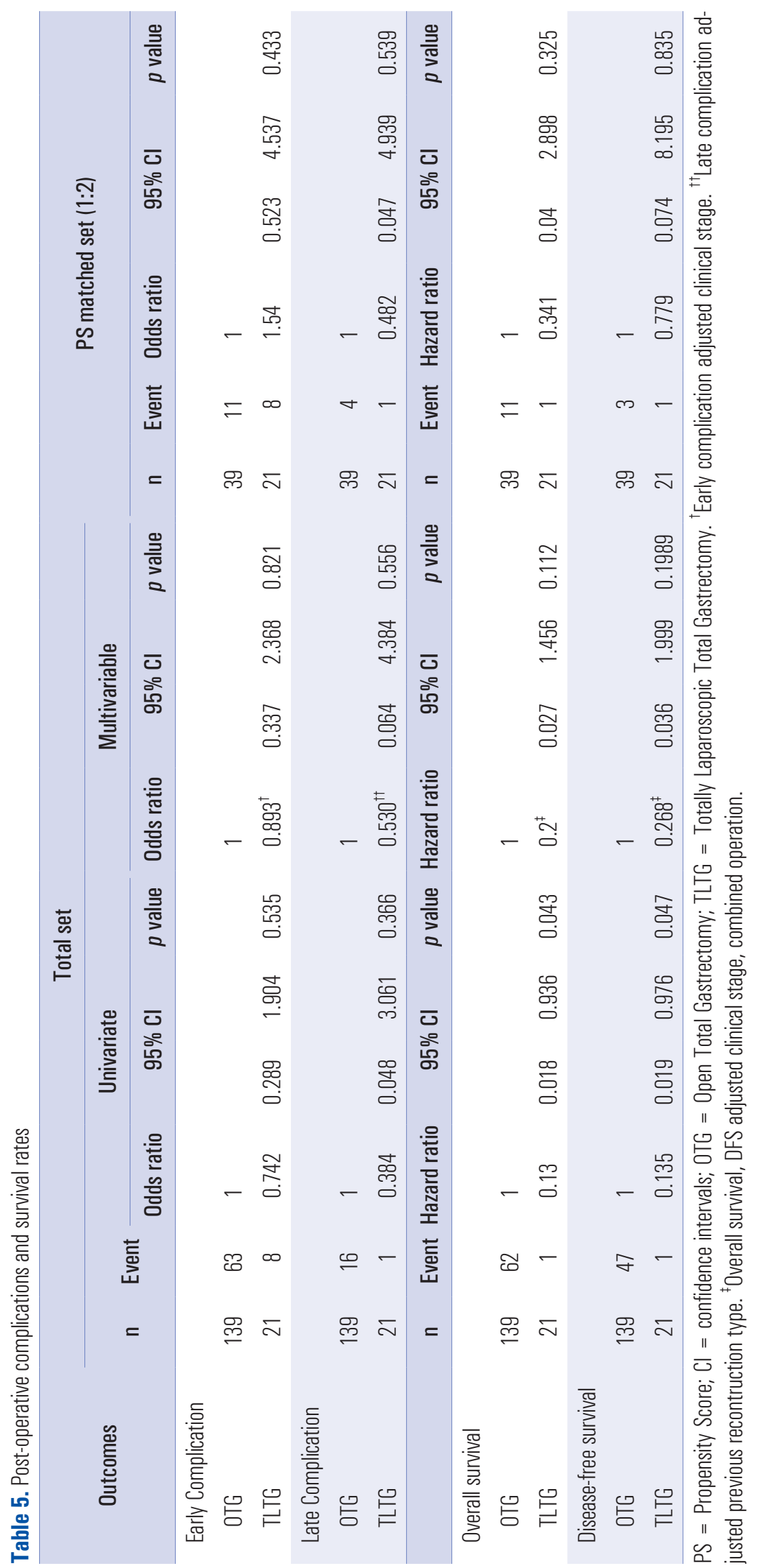


groups in the total set (before PSM) were 62.1\% and 93.3\%, respectively, ( $p=0.017$ by log-lank test) and their 5-year DFS rates in the total set (before PSM) were 59.5\% and 92.3\%, respectively ( $p=0.015$ by log-lank test). The 5 -year OS rates of the OTG and TLTG groups in the PS matched set were $86.2 \%$ and $93.3 \%$, respectively, ( $p=0.325$ by log-lank test) and their 5-year DFS survival rates in the PS matched set were $82 \%$ and $92.3 \%$, respectively ( $p=0.835$ by log-lank test). After PSM, Table 5 revealed no significant difference between the two groups in terms of OS (HR 0.341, 95\% CI 0.040 2.898, $p=0.325$ ) or DFS rates (HR 0.779, 95\% CI 0.074 8.195, $p=0.835$ ).

\section{DISCUSSION}

The main difficulty in the laparoscopic approach for RGC is the need for a meticulous dissection of the intra-abdominal adhesions between surrounding organs such as the left lateral liver segment, gall bladder, ventral area of the pancreas, lesser curvature of the remnant stomach and the celiac artery. Adhesiolysis in this area should be carefully performed in order to avoid any potential iatrogenic injury such as severe bleeding and gastrointestinal perforation. Therefore, researchers have suggested that the type of preceding surgery (open gastrectomy, cancer surgery, or GD) should be considered when selecting potential cases for the laparoscopic approach in RGC, ${ }^{6,13,14}$

In our study, there were several differences in baseline $\mathrm{pa}^{-}$ tient characteristics between the two groups prior to PSM. GD was more common in the TLTG group; this was presumably just a matter of chance because of the retrospective nature of the study. The OTG group contained a higher proportion of patients with advanced clinical tumor stage and previous open gastrectomies, which was preferably selected with a less difficult open approach in the early period before the learning curve of LG. No combined major operation was performed in the TLTG group because other major surgeries require an open approach. In the early period, some factors such as clinical stage, and previous gastrectomy type did influence our choice of operation. However, TLTG in RGC is lately being performed more widely lately, since these factors have been overcome as a result of surgical experience and recent technique developments. Nowadays only the need for a combined major operation affects the choice of surgical approach because combined major surgery still does requires open surgery.

Our result indicated that the mean operative time was shorter in the OTG group, which may be due to the fact that TLTG is more difficult to manipulate, and dissections need to be performed with extreme care in LG. Postoperatively, bowel movement recovered at a faster reate in the TLTG group, which is due to the fact that laparoscopic surgery requires less surgical manipulation. All other postoperative surgical outcomes such as blood loss, operative events, pain score and post-operative hospital stay, and complications were not significantly different between the two groups. Previous studies have reported that the overall surgical complication rate was $19 \sim 47 \%$ and the surgery-related mortality rate was $2 \sim 13 \%$ in $R G{ }^{13,15-19}$ We observed an overall complication rate of $38.1 \%$ in the early period and $4.76 \%$ in the late period after TLTG (not much higher than in previous studies) and a mortality rate of $0 \%$. Therefore, these findings suggest that this method of TLTG is feasible and safe in RGC. In our study, intra-operative events associated with adhesiolysis included bleeding (8 cases), major vessel injury ( 2 cases), and bowel injury (3 cases) in the OTG group. In the TLTG group, there was one case of duodenal wall injury. All intraoperative events in both groups were successfully managed (primary repair, splenectomy, colectomy, etc.) during operation without leading to postoperative complications. Some investigators have reported that internal herniation develops more frequently in TLTG than OTG, ${ }^{20-22}$ but we encountered no internal herniation postoperatively in either group. The reason may have been the closure of the mesenteric defect during surgery and the formation of more adhesions due to the re-operation for RGC.

The standard treatment for RGC, like for gastric cancer, is radical gastrectomy including D2 lymph node dissection. ${ }^{16,23-25}$ We found no difference in the number of retrieved lymph nodes between OTG and TLTG in either the total set or the PS matched set (Table 2). There was no difference in OS or DFS between the OTG and TLTG group after PSM (Table 5) indicating that TLTG is oncologically safe and feasible.

Intracorporeal reconstruction in LG has several benefits, such as improved cosmetic outcomes, decreased pain, faster recovery, and decreased postoperative complications; hence there is a trend for the intracorporeal method in LG to be used more widely for the treatment of gastric cancer. ${ }^{9,25-28}$ However, the manipulations involved in TLTG require more experienced surgical skill for both surgeon and assistants due to the complexity associated with the reconstruction of anastomosis, and TLTG after RGC demands even greater surgical skill. Despite the difficulty in performing TLTG for RGC, the laparoscopic approach allowed us to have a broader view of the surgical field and to identify surgical structures finely. This made it easier to access deeper organ regions and enabled surgeons to perform meticulous dissections and reconstructions of EJ. The reduced bowel manipulation and accurate dissection during TLTG may lead to fewer adhesions after TLTG, and make it less difficult to perform meticulous dissection if more surgery is needed in the future; hence the intracorporeal laparoscopic approach may be preferred in any re-operation.

There are some reservations about performing TLTG for RGC. One must be careful not to injure the intestine when 
the first trocar is inserted because of adhesions resulting from the previous operation. If severe adhesions are present in the umbilical area, the first laparoscopic camera port should be inserted through an adhesion-free area. In one case in this study, the camera port was inserted through the right lower quadrant due to severe adhesion in the umbilical area. Careful handling is always needed to reduce operative trauma because laparoscopic instruments can cause accidental intestinal injury when manipulating the intestines. Careful dissection with laparoscopic scissors and an energy device is necessary to prevent intestinal perforation during the lysis of adhesions. The following are key requirements for carrying out safe TLTG: (1) The correct choice of the type of EJ (functional method vs overlapping method) according to the status of the mesentery and esophagus, or proximal resection margin from RGC; (2) Full mobilization of the Roux-en Y limb in order to avoid tension on the EJ; (3) Exposure of the mucosa of the esophageal stump to locate the true lumen of the esophagostomy when inserting the endoscopic linear stapler to form the EJ; (4) Proper counter traction of the esophagus and jejunum during the reconstruction of the common channel so as not to tear the intestine; (5) Careful closure of the entry hole using an endoscopic linear stapler with a full layer capture to prevent the mucosa from slipping out; (6) Closure of the transmesenteric and Petersen's spaces to prevent internal herniation.

This study had several limitations because it was a single center retrospective study, and the number of cases was small because RGC is rare. However, it used the PSM method for adjusting confounding factors to balance the baseline preoperative characteristics that affect the decision between OTG and TLTG. Nevertheless, since after matching the selected study sample predominantly consisted of early gastric cancer, the oncologic safety of TLTG has not been established for AGC. Therefore, a large, randomized and prospective multicenter clinical trial should be considered to provide evidence of the safety and efficacy of TLTG in RGC.

In conclusion, the morbidity and mortality of TLTG were comparable to those of OTG, and the oncologic outcomes were similar, especially in early stage RGC cases. Thus, TLTG may be safe and feasible for early stage RGC, considering the similar surgical outcomes with OTG despite the need for longer operative time for adhesiolysis.

\section{FUNDING}

There are no funding sources to disclose in relation to this study.

\section{REFERENCES}

1) Liao G, Wen S, Xie X, Wu Q. Laparoscopic gastrectomy for remnant gastric cancer: Risk factors associated with conversion and a systematic analysis of literature. Int J Surg 2016;34:17-22.

2) Tanigawa N, Nomura E, Niki M, et al. Clinical study to identify specific characteristics of cancer newly developed in the remnant stomach. Gastric Cancer 2002;5:23-28.

3) Sinning C, Schaefer N, Standop J, Hirner A, Wolff M. Gastric stump carcinoma - epidemiology and current concepts in pathogenesis and treatment. Eur J Surg Oncol 2007;33:133-139.

4) Mezhir JJ, Gonen M, Ammori JB, Strong VE, Brennan MF, Coit DG. Treatment and outcome of patients with gastric remnant cancer after resection for peptic ulcer disease. Ann Surg Oncol 2011;18:670-676.

5) Ohira M, Toyokawa T, Sakurai K, et al. Current status in remnant gastric cancer after distal gastrectomy. World J Gastroenterol 2016; 22:2424-2433.

6) Yamada H, Kojima K, Yamashita T, Kawano T, Sugihara K, Nihei Z. Laparoscopy-assisted resection of gastric remnant cancer. Surg Laparosc Endosc Percutan Tech 2005;15:226-229.

7) Kim HS, Kim BS, Lee IS, Lee S, Yook JH, Kim BS. Laparoscopic gastrectomy in patients with previous gastrectomy for gastric cancer: a report of 17 cases. Surg Laparosc Endosc Percutan Tech 2014;24:177-182.

8) Qian F, Yu PW, Hao YX, et al. Laparoscopy-assisted resection for gastric stump cancer and gastric stump recurrent cancer: a report of 15 cases. Surg Endosc 2010;24:3205-3209.

9) Kim HS, Kim BS, Lee IS, Lee S, Yook JH, Kim BS. Comparison of totally laparoscopic total gastrectomy and open total gastrectomy for gastric cancer. J Laparoendosc Adv Surg Tech A 2013; 23:323-331.

10) Kim HS, Kim BS, Lee S, Lee IS, Yook JH, Kim BS. Reconstruction of esophagojejunostomies using endoscopic linear staplers in totally laparoscopic total gastrectomy: report of 139 cases in a large-volume center. Surg Laparosc Endosc Percutan Tech 2013; 23:e209-216.

11) Kim HS, Kim MG, Kim BS, Yook JH, Kim BS. Totally laparoscopic total gastrectomy using endoscopic linear stapler: early experiences at one institute. J Laparoendosc Adv Surg Tech A 2012; 22:889-897.

12) Dindo D, Demartines N, Clavien PA. Classification of surgical complications: a new proposal with evaluation in a cohort of 6336 patients and results of a survey. Ann Surg 2004;240:205-213.

13) Son SY, Lee CM, Jung DH, et al. Laparoscopic completion total gastrectomy for remnant gastric cancer: a single-institution experience. Gastric Cancer 2015;18:177-182.

14) Nagai E, Nakata K, Ohuchida K, Miyasaka Y, Shimizu S, Tanaka M. Laparoscopic total gastrectomy for remnant gastric cancer: feasibility study. Surg Endosc 2014;28:289-296. 
15) Kwon IG, Cho I, Choi YY, Hyung WJ, Kim CB, Noh SH. Risk factors for complications during surgical treatment of remnant gastric cancer. Gastric Cancer 2015;18:390-396.

16) Ahn HS, Kim JW, Yoo MW, et al. Clinicopathological features and surgical outcomes of patients with remnant gastric cancer after a distal gastrectomy. Ann Surg Oncol 2008;15:1632-1639.

17) Lissens P, Filez L, Aerts R, et al. Surgery for gastric remnant carcinoma following Billroth II gastrectomy. Eur J Surg Oncol 1997; 23:518-521.

18) Kodera Y, Yamamura Y, Torii A, et al. Gastric stump carcinoma after partial gastrectomy for benign gastric lesion: what is feasible as standard surgical treatment? J Surg Oncol 1996;63:119-124.

19) Firat O, Guler A, Sozbilen M, Ersin S, Kaplan H. Gastric remnant cancer: an old problem with novel concerns. Langenbecks Arch Surg 2009;394:93-97.

20) Ojima T, Nakamori M, Nakamura M, et al. Internal Hernia After Laparoscopic Total Gastrectomy for Gastric Cancer. Surg Laparosc Endosc Percutan Tech 2017;27:470-473.

21) Aghajani E, Jacobsen HJ, Nergaard BJ, Hedenbro JL, Leifson BG, Gislason H. Internal hernia after gastric bypass: a new and simplified technique for laparoscopic primary closure of the mesenteric defects. J Gastrointest Surg 2012;16:641-645.

22) Kimura $H$, Ishikawa $M$, Nabae $T$, et al. Internal hernia after laparoscopic gastrectomy with Roux-en-Y reconstruction for gastric cancer. Asian J Surg 2017;40:203-209.
23) Li F, Zhang $\mathrm{R}$, Liang $\mathrm{H}$, et al. A retrospective clinicopathologic study of remnant gastric cancer after distal gastrectomy. Am J Clin Oncol 2013;36:244-249.

24) Thorban S, Bottcher K, Etter M, Roder JD, Busch R, Siewert JR. Prognostic factors in gastric stump carcinoma. Ann Surg 2000; 231:188-194.

25) Kanaji S, Harada H, Nakayama S, et al. Surgical outcomes in the newly introduced phase of intracorporeal anastomosis following laparoscopic distal gastrectomy is safe and feasible compared with established procedures of extracorporeal anastomosis. Surg Endosc 2014;28:1250-1255.

26) Gong CS, Kim BS, Kim HS. Comparison of totally laparoscopic total gastrectomy using an endoscopic linear stapler with laparoscopic-assisted total gastrectomy using a circular stapler in patients with gastric cancer: A single-center experience. World J Gastroenterol 2017;23:8553-8561.

27) Kim HS, Kim MG, Kim BS, et al. Comparison of totally laparoscopic total gastrectomy and laparoscopic-assisted total gastrectomy methods for the surgical treatment of early gastric cancer near the gastroesophageal junction. J Laparoendosc Adv Surg Tech A 2013;23:204-210.

28) Jun G, Ping L, Jie C, Qi L, Tang D, Wang D. Totally laparoscopic vs. laparoscopically assisted distal gastrectomy for gastric cancer: a meta-analysis. Hepatogastroenterology 2013;60:1530-1534. 